\title{
Imaging spectroscopy with the atomic force microscope
}

\author{
David R. Baselt ${ }^{\mathrm{a})}$ and John D. Baldeschwieler \\ Noyes Laboratory of Chemical Physics, California Institute of Technology 127-72, \\ Pasadena, California 91125
}

(Received 15 November 1993; accepted for publication 5 March 1994)

\begin{abstract}
Force curve imaging spectroscopy involves acquiring a force-distance curve at each pixel of an atomic force microscope image. Processing of the resulting data yields images of sample hardness and tip-sample adhesion. These images resemble $Z$ modulation images and the sum of forward and reverse friction images, respectively, and like them exhibit a number of potentially misleading contrast mechanisms. In particular, $X Y$ tip motion has a pronounced effect on hardness images and the meniscus force on adhesion images.
\end{abstract}

\section{INTRODUCTION}

Atomic force microscopy (AFM) operates by measuring attractive or repulsive forces between a tip and the sample. ${ }^{1}$ In its repulsive mode, the instrument lightly touches a tip at the end of a leaf spring or "cantilever" to the sample. As a raster scan drags the tip over the sample, a detection apparatus (the commonly used "optical lever"2 measures the position of a laser spot reflected off the cantilever) measures the vertical deflection of the cantilever, which indicates the local sample height. A feedback mechanism keeps the cantilever deflection constant by adjusting the separation $Z$ between the base of the cantilever and the sample. An image of $Z$ as a function of tip position $X Y$ thus represents sample topography. On some samples AFMs can obtain such topographic images at atomic resolution.

If the sample moves perpendicular to the cantilever, friction between the tip and the sample creates lateral forces that twist the cantilever. Force microscopists have devised various methods to measure this torsion and thus provide friction or "lateral deflection" images."

Differential lateral deflection (DLD) images, the sum of forward and reverse friction images, generally provide more useful data than raw friction images. "4,5 "Forward" and "reverse" refers to the scan direction: For each scan line the tip first moves left to right, the data going into the forward image; the tip then retraces its path, moving right to left, the data going into the reverse image. The SPM software that we use multiplies all forward friction data by -1 so that sticky areas appear bright in both images; thus, unless otherwise specified, references to forward friction data will assume that the data have becn multiplied by -1 . Addition of the thusprocessed forward and reverse images (or subtraction of the unprocessed images) creates a DLD image.

AFMs can measure sample hardness by pressing the tip into the sample at each data point and determining the change in cantilever deflection. ${ }^{6}$ Our instrument obtains such " $Z$ modulation images" by adding a sinusoidal modulation to $Z$ and measuring the resulting modulation amplitude of the cantilever by lock-in detection. ${ }^{7}$

a) Current address: Department of Biology, Yale University, KBT 338, P.O. Box 208103, New Haven, CT 06520.
Similarly, AFMs can obtain "force spectra" or "force curves" by increasing or decreasing $Z$ over a large range while continuously recording cantilever deflection. This process generates graphs that bear a passing resemblance to Lennard-Jones force curves but differ fundamentally: AFM force curves show how the tip-sample interaction force varies with separation between the base of the cantilever and the sample, while an equivalent Lennard-Jones force curve would show how it varies with separation between the tip and sample. Previous applications of force curves have included measuring the mechanical properties of materials, 8,9 studying electrostatic, hydration, and other forces between surfaces in solution and in air, ${ }^{10,11}$ directly observing colloidal forces, ${ }^{12}$ and investigating the properties of thin films. ${ }^{13-17}$

Figure 1 shows a representative AFM force curve. The distance $Z$ between the sample and the base of the cantilever increases from the left- to the right-hand side. At the lefthand edge of the curve, the tip and sample touch. As $Z$ increases, the force on the sample (cantilever deflection) decreases, eventually becoming negative. When imaging in air, a layer of water condensation and other contamination covers both the tip and sample, forming a meniscus that pulls the two together. ${ }^{11}$ 'Thus, the cantilever has to exert an upward (negative) force to pull the tip free of the meniscus. The lowest point of the force curve indicates the strength of the meniscus attraction, $2 \mathrm{nN}$ in this case, although $10-100 \mathrm{nN}$ is more typical. Having pulled free of the sample, the cantilever returns to zero deflection and remains there as $Z$ continues to increase (for an analysis of both the repulsive and attractive regions of force curves see Refs. 8-9).

"Imaging spectroscopy" involves taking hundreds or thousands of spectra over the course of a raster scan. This technique provides a spectrum at every pixel of the image. To date, imaging spectroscopy has primarily been performed with scanning tunneling microscopy (STM), which operates by inducing and measuring a tunneling current between a conductive tip and the sample. Imaging spectroscopy with the STM may, for example, involve obtaining tunneling current versus tip or sample bias spectra in a technique dubbed "current imaging tunneling spectroscopy" (CITS).$^{18}$ Mate and co-workers ${ }^{16}$ have performed a one-dimensional form of imaging spectroscopy with AFM, taking a few hundred force curves as the tip scanned along a line. Miyamoto and 


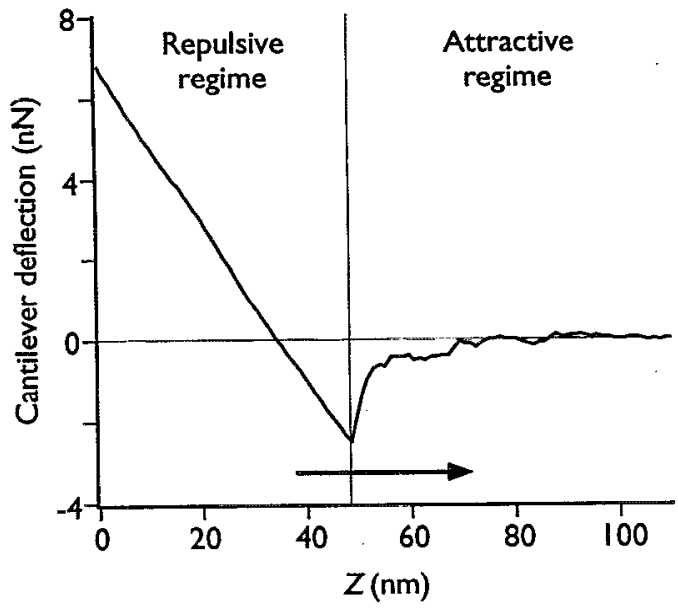

FIG. 1. Force curve acquired in air. At $Z=0 \mathrm{~nm}$ the cantilever pushes down on the tip (positive force), and tip and sample contact each other (repulsive regime). As $Z$ increases, the cantilever exerts less force and then begins to pull up on the tip (negative force). Eventually the cantilever exerts enough force to pull the tip free of the meniscus ( $2 \mathrm{nN}$ in this case, an unusually low figure). After this point, only attractive forces affect the cantilever deflection (attractive regime).

co-workers ${ }^{17}$ have used force curve imaging spectroscopy to characterize thin-film disk media.

We discuss here our implementation of "force curve imaging spectroscopy" on the AFM and provide interpretations of the resulting images, with an emphasis on artifacts and alternative methods of obtaining similar data.

\section{METHODS}

We obtained all images on a scanned-cantilever AFM stage $^{19}$ with a digital signal processor-based control system. ${ }^{7}$ The stage acquires topography and friction data simultaneously, using an optical lever equipped with a four-segment photodiode. ${ }^{3}$ The imaging spectroscopy acquisition and analysis software runs on the digital signal processor, which we programmed in C (TMS320C30 C compiler, Texas Instruments, Richardson, TX). Topometrix, Inc. (Santa Clara, CA) supplied the digital-analog converter hardware (TMX2000 electronic control unit). We used 0.6- $\mu \mathrm{m}$-thick, V-shaped, pyramidal tipped silicon oxynitride cantilevers obtained from Park Scientific Instruments (Mountain View, CA). These cantilevers have a manufacturer-estimated spring constant of $0.37 \mathrm{~N} / \mathrm{m}$. The quantitative measurements of friction and adhesion presented below are based on this value and should be regarded as order-of-magnitude estimates. ${ }^{20}$

The force curve acquisition software works as follows (ramp times and other imaging parameters are those used for this article; different instruments and samples may require different parameters). Before each force curve the software moved the tip to the appropriate $X Y$ location over a $0.5 \mathrm{~ms}$ period, with $Z$ under feedback control. For each force curve the software turned the feedback off, then ramped $Z$. over a 1 $\mathrm{ms}$ period to the starting value of the force curve. After a 0.5 ms pause, the software acquired a 64 point force curve by ramping $Z$ to its final value over a period of $32 \mathrm{~ms}$. Finally,

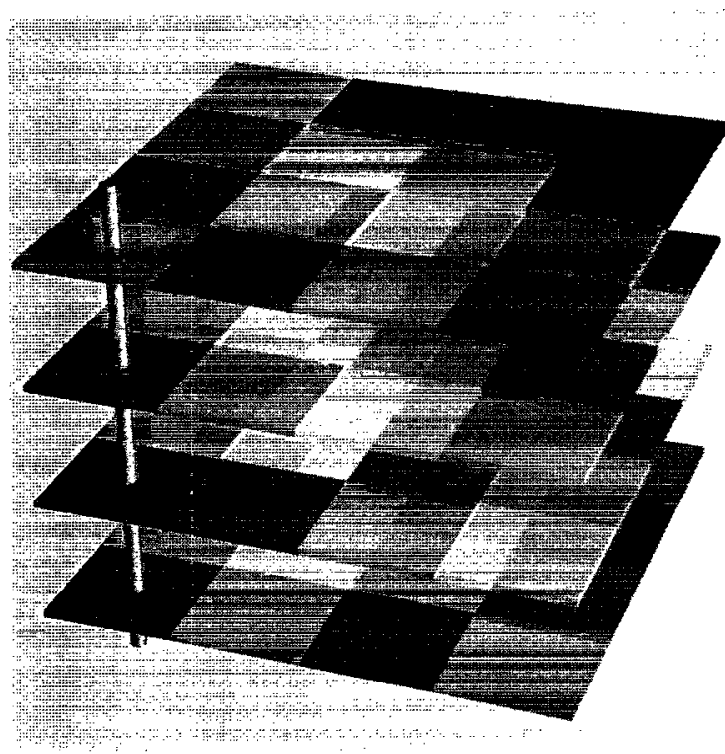

FIG. 2. Schematic of a $4 \times 4 \times 4$ imaging spectroscopy stack. Each of the four slices (horizontal planes) is a $4 \times 4$ image of cantilever deflection at a different $Z$. Each force curve (the vertical rod indicates one) shows how cantilever deflection depends on $Z$ at a given location on the sample.

it ramped $Z$ back to its most recent feedback-determined value over a $0.5 \mathrm{~ms}$ period, restarted the feedback, and began the cycle again. Our imaging spectroscopy stacks generally contain $64 \times 64 \times 64$ data points, and each force curve has a $1.2 \mu \mathrm{m} Z$ range.

All images represent raw data except as specified. We generated the "slope of topography" image in Fig. 4 by replacing each data point with the difference between it and the point immediately to its left-hand side.

We prepared samples of rat-tail (type I) collagen by removing the skin from frozen rat tails, shaving off a slice of the underlying tendon, and cutting or tearing the slice with two razor blades on a standard glass microscope slide. A number of microscopic collagen fibrils generally adhere to the slide near the location of the cut; we removed the macroscopic remnants of the collagen slice manually. Before using, we washed the slide with $5 \mathrm{~mL} \mathrm{1:100}$ Triton X-100 followed by a $30 \mathrm{~s}$ rinse in flowing water and an air-drying period of at least $1 \mathrm{~h}$. We imaged the fibrils in air.

\section{RESULTS}

Imaging spectroscopy creates a three-dimensional "stack" of two-dimensional images or "slices" (Fig. 2). Each slice is an image of cantilever deflection at a specific value of $Z$. The user can display individual slices or inspect the force curve for any given point of a slice.

Figure 3 shows a typical force curve acquired in air. Unlike the idealized curve in Fig. 1, Fig. 3 exhibits "clipping" caused by the limited digitization range of our analogto-digital converters. The data interpretation software must be able to distinguish such invalid, clipped readings of cantilever deflection from valid readings. Furthermore, a cantilever deflection sensor output of $0 \mathrm{~V}$ does not necessarily correspond to zero cantilever deflection. 


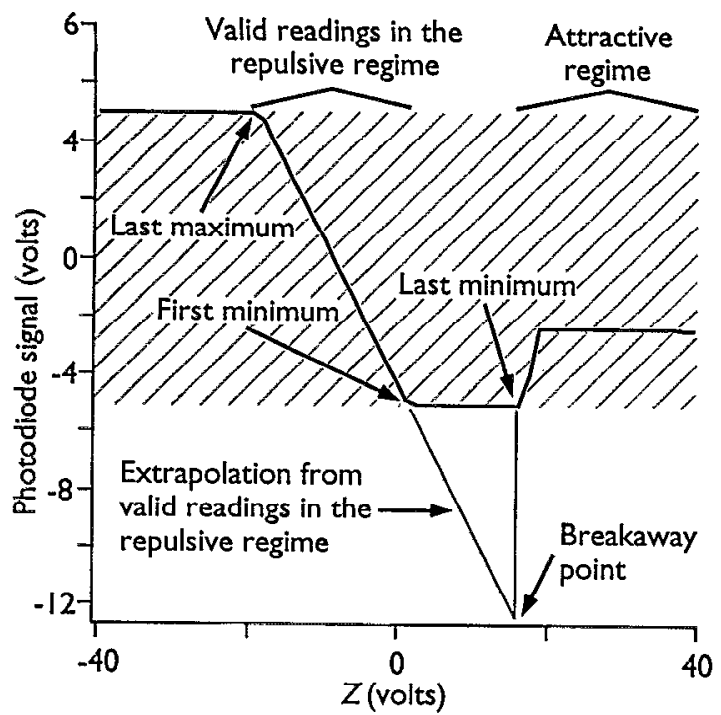

FIG. 3. Force curve taken in air. Photodiode signal is proportional to cantilever deflection. Force curves such as this often exceed the detection range of the AFM (cross hatched). In order to determine sample hardness and tip-sample adhesion, the AFM software isolates the valid (within the detection range) repulsive-regime portion of the curve and fits a line to it. Operationally, the software defines this region as the portion of the curve between the last occurrence of the maximum photodiode signal $(5 \mathrm{~V}$ in this case) and the first occurrence of the minimum $(-5 \mathrm{~V})$. The slope of this line indicates sample hardness, while its $Y$ intercept with the tip-sample breakaway point (the last occurrence of the $-5 \mathrm{~V}$ minimum) yields the adhesion force. $Z$ range: $1.2 \mu \mathrm{m}$.

Imaging spectroscopy can be performed in either "relative" or "absolute" modes. In either case, the user specifies minimum and maximum $Z$ values for the spectra. In the relative mode, the software adds these values to the feedbackdetermined $Z$ before taking each spectrum, while in the absolute mode, the software acquires all spectra between the same two values of $Z$. Relative-mode slices superficially resemble error signal images, showing the slope of topography, while absolute-mode slices resemble topography images (Fig. 4). We have found relative spectroscopy to be the more useful mode, since overall sample tilt tends to produce excessive clipping of absolute-mode spectra.

Relative-mode slices exhibit their poorest contrast at $Z=0$; contrast improves the more $Z$ deviates from its feedback-determined value. Furthermore, the contrast inverts as $Z$ passes through 0 . Absolute-mode slices do not exhibit this type of contrast variation.

Since individual slices or force curves are generally difficult to interpret, we have found it helpful to replace each force curve with a relevant variable mcasured from it, thereby reducing stacks to single images. For example, the slope of a force curve in the repulsive regime is, to a first approximation, proportional to the hardness of the sample. Thus, to estimate sample hardness from a force spectrum, the software isolates the segment of the spectrum corresponding to valid repulsive-regime measurements (Fig. 3). It then fits a line to that segment by linear regression and stores the slope of that line. Repeating this process at each pixel of the force spectroscopy stack produces an image of sample hardness

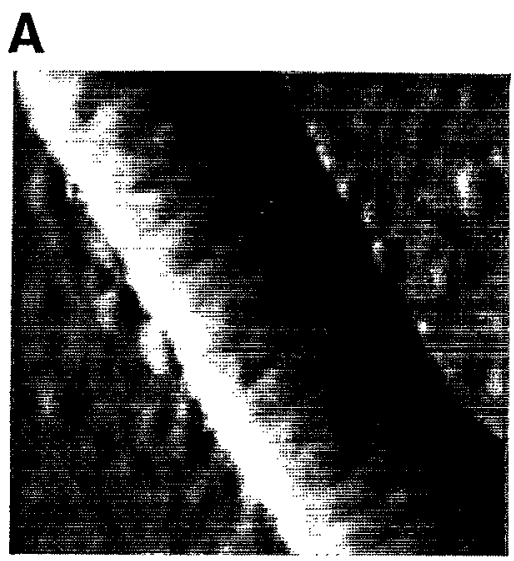

B
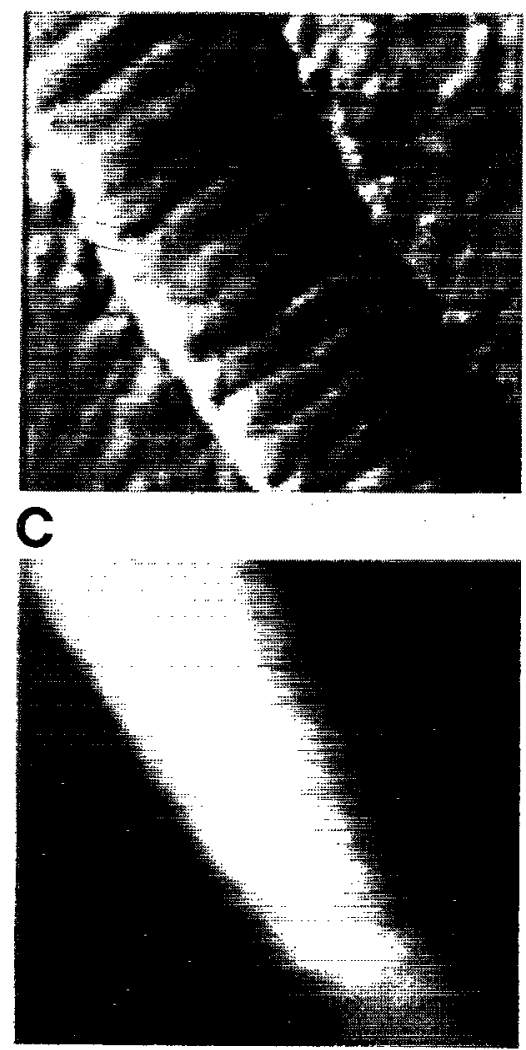

FIG. 4. (A) $730 \times 730 \mathrm{~nm}$ slope-shaded topographic image of two collagen fibrils lying one on top of the other, with slices from (B) $700 \times 700 \mathrm{~nm}$ relative and (C) $730 \times 730 \mathrm{~nm}$ absolute force curve imaging spectroscopy stacks. Note the extensive clipping (solid white or black areas) on the absolute-mode slice. All stacks $64 \times 64 \times 64$ points.

[Fig. 5(A)]. Such images bear a strong resemblance to $Z$ modulation-derived hardness images [Fig. 5(B)].

Similarly, the difference between the cantilever deflection just before and just after pulling away from the sample provides a measurement of tip-sample adhesion. Since the former point-the lowest point of the force curve-is often clipped by the analog-to-digital converter, the software estimates its position by extrapolating a line fit to the repulsive regime (Fig. 3). Repeating this calculation at each spectrum yields an adhesion image [Fig. 6(A)] that closely resembles DLD inages of the same area [Fig. 6(B)]. 
A

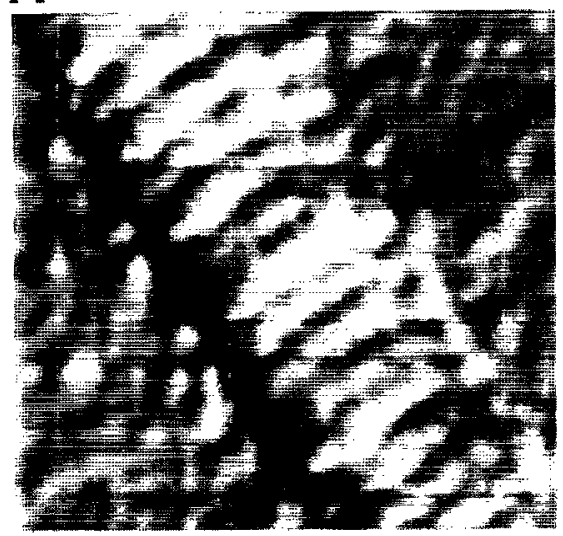

B

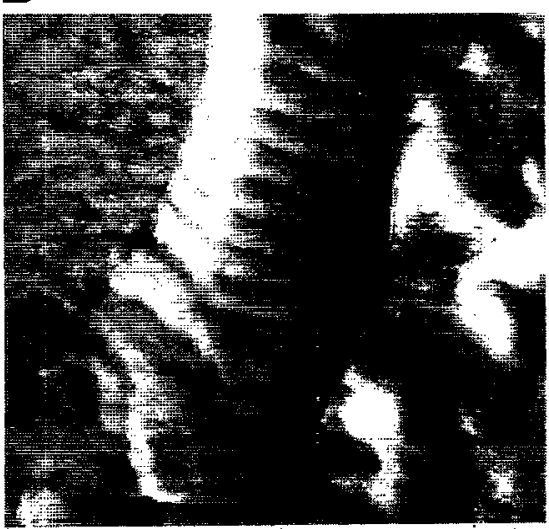

FIG. 5. (A) $700 \times 700 \mathrm{~nm}$ hardness image derived from the relative-mode imaging spectroscopy stack in Eig. 3. $64 \times 64$ points. (B) $1.0 \times 1.0 \mu \mathrm{m} \mathrm{Z}$ modulation hardness image of a similar sample. $250 \times 250$ points. 'l'he $Z$ modulation-derived image is sharper because of its higher pixel density.

Relative and absolute mode stacks yield similar hardness and adhesion images.

\section{DISCUSSION}

\section{A. Contrast varlabllity of relative-mode slices}

Relative-mode slices have low contrast near $Z=0$ because of the influence of the feedback loop. In relative mode, $Z=0$ is the value of $Z$ set by the feedback loop, which exists to keep cantilever deflection constant. Therefore, it is reasonable that an image of cantilever deflection should exhibit a minimum of point-to-point variability when $Z=0$. As $Z$ deviates from 0 , cantilever deflection changes at a rate that depends on the apparent hardness of the sample. Since this varies between different locations, contrast increases.

\section{B. Hardness images}

It is not surprising that spectroscopy- and modulationderived hardness images resemble each other, since both represent measurements of the slope of the repulsive-regime force curve. The two types of image measurements thus exhibit the same contrast mechanisms and artifacts.

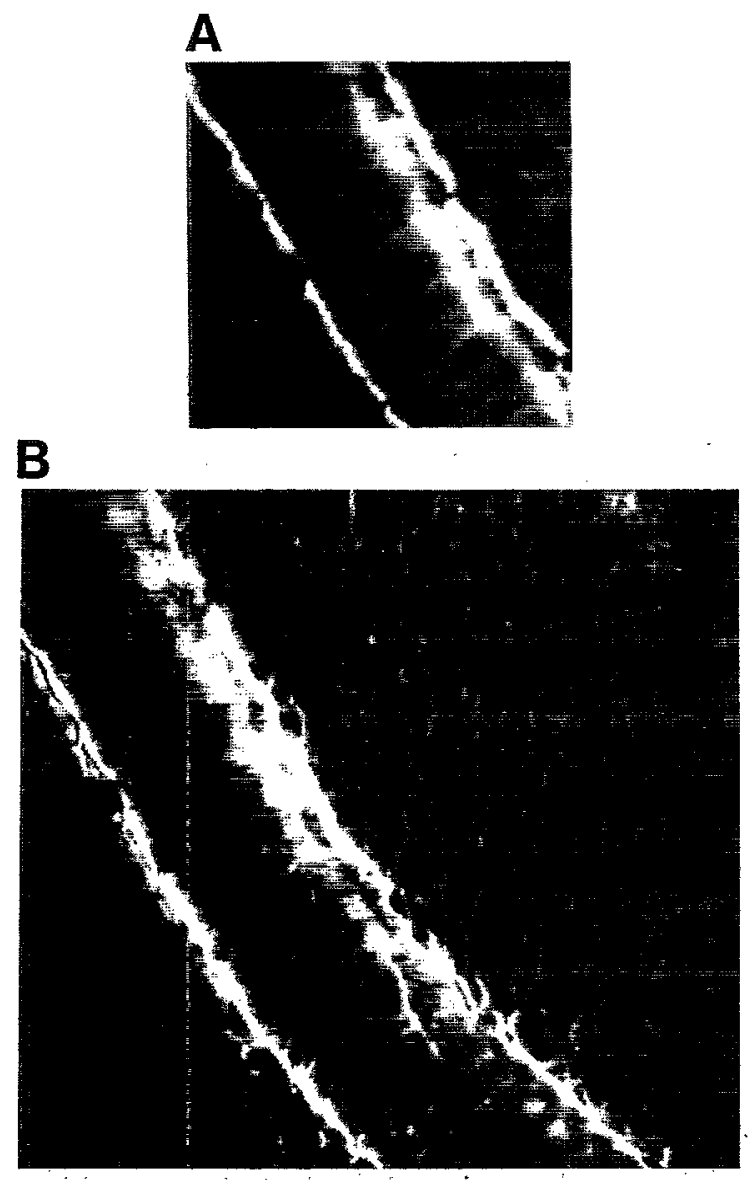

FIG. 6. (A) $700 \times 700 \mathrm{~nm}$ adhesion image derived from the relative-mode imaging spectroscopy stack in Fig. 3. Average adhesion is $55 \mathrm{nN}$, increasing to $120 \mathrm{nN}$ at the edge of the collagen fibril. $64 \times 64$ points. (B) $1.3 \times 1.3 \mu \mathrm{m}$ DLD image of the same region. Average frictional force is $13 \mathrm{nN}$, increasing to $37 \mathrm{nN}$ at the fibril edges. $250 \times 250$ points. The greater resolution of the DLD image is due to its greater pixel density.

The features observed in Figs. 5(A) and 5(B) are an artifact of the imaging process that we refer to as "tip sliding." Collagen exhibits negligible elasticity when dry; only when submerged in water do the fibrils become soft. ${ }^{5}$ Hardness images of negligibly elastic samples, such as Figs. 5(A) and 5(B), often resemble slope-shaded topography imagesapparent hardness varies as a roughly linear function of sample slope [Fig. 7(A)]. This artifact arises because the lateral force on the tip can change during the $Z$ modulation associated with both types of hardness measurements. The sample can move laterally under the tip if the modulation is not perpendicular to the cantilever; and if the sample surface is not parallel to the cantilever, lateral forces exist that cause the tip to slide down the slope. In either case, the tip movement reduces the amount of cantilever deflection induced by changing $Z$, thus making the sample appear softer than it actually is. The amount of tip movement increases with the slope of the sample.

In many AFMs (including our own) the cantilever is placed at a $5^{\circ}-10^{\circ}$ angle to the $Z$ axis. The sample then has an overall tilt relative to the cantilever, and the modulation direction is not perpendicular to the cantilever, giving rise to 

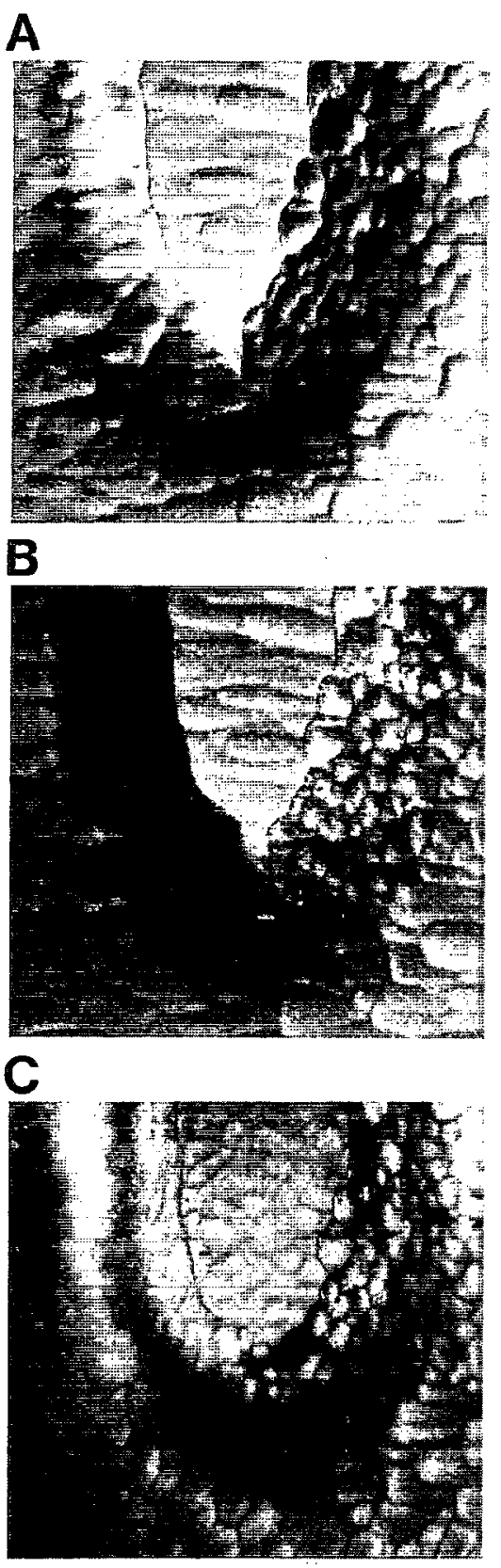

FIG. 7. Changing the $Z$ modulation angle to correct for overall sample slope can alleviate the tip sliding artifact in hardness images. These are $630 \times 630$ $\mathrm{nm} Z$ modulation-derived hardness images of a compact disk pit. (A) Image with no adjustments to the modulation angle. (B) Changing the modulation angle can reverse the slope-shaded appearance of an elasticity image, confirming that tip sliding causes this artifact. (C) In the properly corrected image, slope artifacts still appear, but with greatly reduced magnitude (not visible because we have increased the contrast correspondingly). The corrected image resembles a light-shaded image with the light source directly above it. Also note the smearing of the features when $Z$ modulation is not perpendicular to the sample. $10 \mathrm{~nm} Z$ modulation amplitude. All images have $250 \times 250$ points.

the light-shaded appearance. Nonorthogonality of the scanner axes compounds the problem with some instruments. The software can correct for overall tilt and nonorthogonality by adding some of the $Z$ signal into $X$ and $Y$ (Figs. 7(B) and $7(\mathrm{C})$ ], thereby changing the modulation angle so it is perpen- dicular to the sample. In practice, we set the angle for each sample based on a visual inspection of hardness images. This alleviates but does not eliminate tip sliding. In order to completely eliminate slope artifacts, the software would have to automatically determine the modulation angle at each pixel by searching for the angle that yields the greatest hardness measurement.

Hoh and Engel ${ }^{21}$ have previously described a related force curve artifact. When acquiring force curves in two directions, both pulling the tip and sample apart and bringing them together, they note that the repulsive-regime portions of the two curves are offset from (and parallel to) each other. The amount of offset varies with the rate of tip-sample approach and separation. The authors attribute this hysteresis to cantilever bowing induced by lateral forces on the tip, and suggest that it might be minimized by modulating the sample perpendicular to the cantilever.

Thus, $Z$ modulation can result in varying lateral forces on the tip, inducing both tip sliding and cantilever bowing, and thereby altering both the slope and offset of force curves.

It is also important to note that apparent sample hardness also varies with tip shape, amount of force on the sample, and the presence of contaminant films. ${ }^{14,15}$

\section{Adhesion images}

All the features seen in Fig. 6 result from meniscus forces. Although we have observed "sticky regions" on collagen fibrils that do not result from meniscus forces, ${ }^{5}$ these features only appear when the tip is sharp in relation to the topography it is imaging, and the tip used for Fig. 5 and 6 is comparatively dull. Although we have not quantitated the radius of this particular tip, the image quality is consistent with a tip radius of hundreds of nanometers; a good tip would have a radius of tens of nanometers.

Note that adhesive forces are high in the grooves of the collagen fibril and low on the ridges (Fig. 6). This contrast arises because meniscus attraction increases with increasing tip radius. ${ }^{22}$ Topologically, a tip resting in a groove is equivalent to a larger-radius tip on a flat surface; the effective tip radius thus increases when the tip passes over a groove or any other surface feature (such as a step or a dimple) that curves up toward the tip. Thus, we should expect to observe increased adhesion in the grooves as well as along the edges of the collagen fibril. Similar logic demonstrates that adhesion should decrease when the tip passes over ridges. Tipsample adhesion thus depends in part on the curvature, or the second derivative, of topography.

To explain why differential lateral deflection images resemble adhesion images, we consider the various contrast mechanisms observed in friction images and how these mechanisms depend on scan direction. First, friction depends on slope in a direction-dependent manner. Because the cantilever exerts a downward pressure on the tip, lateral force on the cantilever increases when the tip scans up a slope. Since lateral force decreases when the tip scans in the opposite direction, down the slope, this slope dependence cancels out in DLDs.

Second, optical-lever AFMs tend to suffer from artifacts that presumably arise because stray light reflected from the 
sample interferes with light reflected from the cantilever. As a result the raw friction signal varies with $X, Y$, and $Z$ in a direction-independent manner; however, when the AFM software multiplies the forward friction image by -1 , these artifacts become direction dependent and thus cancel out in DLDs. $^{23}$

Third, friction depends on tip-sample adhesion-both long-range attraction such as meniscus forces and van der Waals forces, and forces acting at the point where tip and sample contact. This contrast mechanism does not depend on scan direction; sticky areas appear sticky no matter which way the tip is moving. Thus, it does not cancel out in DLDs.

Therefore, of the three contrast mechanisms found in friction images, DLDs only preserve adhesion. DLDs are thus adhesion images, and should be expected to resemble imaging spectroscopy-derived adhesion images. In both the DLDs and the spectroscopy-derived adhesion images that we present here, most of the observed contrast arises from meniscus forces acting on the body of the tip rather than chemical interactions acting on the tip where it touches the sample.

\section{CONCLUSION}

An important result of the above work is the confirmation that DLD images can provide adhesion data. In addition, recall that $Z$ modulation images provide the same data as spectroscopy-derived hardness images. Both DLD and $Z$ modulation images require considerably less time and computer memory than force curve imaging spectroscopy, and thus can be acquired at greater pixel densities. On the other hand, imaging spectroscopy offers quantitative adhesion measurements, which DLDs do not; it also offers the potential to observe nonlinear elastic behavior, which $Z$ modulation images cannot. By moving the tip into the sample instead of pulling it away, imaging spectroscopy can also provide more detailed information about attractive-regime tip-sample interactions.

Note added in proof. Another implcmentation of imaging spectroscopy is described in an article currently in press (M. Radmacher, J. P. Cleveland, M. Fritz, H. G. Hansma, and P. K. Hansma, Biophys. J.).

\section{ACKNOWLEDGMENTS}

This work was supported in part by a grant from Ford Motor Company and a NSF predoctoral fellowship (D.B.). We thank Topometrix, Inc. for the use of the TMX2000 electronic control unit and its associated computer hardware.

${ }^{1}$ G. Bennig, C. F. Quate, and Ch. Gerber, Phys. Rev. Lett. 56, 930 (1986).

${ }^{2}$ S. Alexander, L. Hellemans, O. Marti, J. Schneir, V. Elings, P. K. Hansma, M. Longmiro, and J. Gurley, J. Appl. Phys. 65, 164 (1989).

${ }^{3}$ G. Meyer and N. M. Amer, Appl. Phys. Lett. 57, 2089 (1990).

${ }^{4}$ D. R. Baselt and J. D. Baldeschwieler, J. Vac. Sci. Technol. B 10, 2316 (1992).

${ }^{5}$ D. R. Baselt, J. P. Revel, and J. D. Baldeschwieler, Biophys. J. 65, 2644 (1993).

${ }^{6}$ M. Radmacher, R. W. Tillmann, and H. E. Gaub, Biophys. J. 64, 735 (1993).

${ }^{7}$ D. R. Baselt, S. M. Clark, M. G. Youngquist, C. F. Spence, and J. D. Baldeschwieler, Rev. Sci. Instrum. 64, 1874 (1993).

${ }^{8}$ N. A. Burnham and R. J. Colton, J. Vac. Sci. Technol. A 7, 2906 (1989).

${ }^{9}$ N. A. Burnham, R. J. Colton, and H. M. Pollock, J. Vac. Sci. Technol. A 9, 2548 (1991).

${ }^{10}$ See, for example, J. H. Hoh, J. P. Cleveland, C. B. Prater, J.-P. Revel, and P. K. Hansma, J. Am. Chem. Suc. 114, 4917 (1992); S. J. O'Shea, M. E. Welland, and T. Rayment, Appl. Phys. Lett. 60, 2356 (1992); Y.-H. Tsao, D. F. Evans, and H. Wennerström, Science 262, 547 (1993); A. L. Weisēnhorn, P. Maivald, H.-J. Butt, and P. K. Hansma, Phys. Rev. B 45(19), 11226 (1992); and see Ref. 11.

${ }^{11}$ A. T. Weisenhorn, P. K. Hansma, T. R. Albrecht, and C. F. Quate, Appl. Phys. Lett, 54, 2651 (1989).

${ }^{12}$ W. A. Ducker, T. J. Senden, and R. M. Pashley, Nature 353, 239 (1991).

${ }^{13}$ See, for example, S. A. Joyce, R. C. Thomas, J. E. Houston, T. A. Michalske, and R. M. Crooks, Phys. Rev. Lett. 68, 2790; and see Refs. 14-17.

${ }^{14}$ G. S. Blackman, C. S. Mate, and M. R. Philpott, Phys. Rev. Lett. 65, 2270 (1990).

${ }^{15}$ N. A. Burnham, D. D. Dominguez, R. L. Mowery, and R. J. Colton, Phys. Rev. Lett. 64, 1931 (1990).

${ }^{16}$ C. M. Mate, M. R. Lorentz, and V. J. Novotny, J. Chem. Phys. 90, 7550 (1989).

${ }^{17}$ T. Miyamoto, R. Kaneko, and Y. Ando, J. Tribology 112, 567 (1990).

${ }^{18}$ R. J. Hamers, R. M. Tromp, and J. E. Demuth, Phys. Rev. Lett. 56, 1972 (1986).

${ }^{19}$ D. R. Baselt and J. D. Baldeschwieler, Rev. Sci. Instrum. 64, 908 (1993).

${ }^{20} \mathrm{~J}$. P. Cleveland, S. Manne, D. Bocek, and P. K. Hansma, Rev. Sci. Instrum. 64, 403 (1993).

${ }^{21}$ J. H. Hoh and A. Engel, Langmuir 9, 3310 (1993).

${ }^{22}$ J. N. Israelachvili, Intermolecular and Surface Forces (Academic, San Diego, 1985).

${ }^{23}$ D. R. Baselt, Ph.D. thesis, California Institute of Technology, Pasadena, CA, 1993. 